\title{
A CLASS OF IDEALS OF THE CENTRE OF A GROUP RING
}

\author{
MICHAEL F, O'REILLY
}

(Received 24 September 1979; revised 3 November 1980)

Communicated by $\mathbf{H}$. Lausch

\begin{abstract}
Reynolds (1972), using character-theory, showed that the $p$-section sums span an ideal of the centre $Z(k G)$ of the group algebra of a finite group $G$ over a field $k$ of characteristic dividing the order of $G$. In O'Reilly (1973) a character-free proof was given. Here we extend these techniques to show the existence of a wider class of ideals of $Z(k G)$.
\end{abstract}

1980 Mathematics subject classification (Amer. Math. Soc.): 16 A 26, 20 C 05, 20 C 20.

\section{Introduction and notation}

Let $G$ be a finite group, $J G$ the group ring over the integers $J$, with centre $Z(J G)$. For $X \subset G$ write $\bar{X}=\Sigma_{g \in X} g$; for $L<G, K<\Re(X) \cap L$ (the normalizer of $X$ in $L$ ) let $\bar{X}_{K}^{L}=\Sigma_{g \in \Omega} \bar{X}^{g}$ where $\Omega$ is a transversal of $K$ in $L$. In particular $\bar{X}_{K}^{G} \in Z(J G)$ and a conjugacy class sum is of the form $b_{C}^{G}$ where $C=C(b)$ is the centralizer of $b$.

The main result is

TheOREM 1. Let $n$ be a fixed divisor of $|G|, L$ a fixed subgroup of $G$. The subspace $\mathscr{W}(L, n)$ of $Z(J G)$ spanned by the set $\left\{(\bar{H} y)_{N}^{G} / H<L, y \in \Re(H)\right.$, $H<N<\Re(H y), N: H$ divides $n\}$ is an ideal of $Z(J G)$.

The ideal $\mathscr{W}(L, n)$ will thus include integer multiples $|C(b)| b_{C(b)}^{G}$ of conjugacy class sums (taking $N=H=\{1\}$ ) but will only include the class sum itself if $|C(b)|$ divides $n$ (taking $H=\{1\}, N=C(b)$ ). 
By extending the ring of coefficients to the $p$-adic integers and mapping canonically to $Z(k G), k$ the residue class field of characteristic $p$, we obtain ideals $\mho^{\prime}(L, n)$ of $Z(k G)$. In the special case where $n=p^{\alpha}$ the generating set may be restricted [Theorem 2] to elements where $N$ is a Sylow $p$-subgroup of $\Re(H y)$. When $|L|=p^{\beta}$ Theorem 3 shows that a further restriction to subgroups $H$ which lie in the Sylow $p$-subgroup of the centralizer of the $p$-regular part of $y$ is permissible. The ideal of $p^{\prime}$-sections is then $W^{\prime}(P, 1)$ where $P$ is a Sylow $p$-subgroup.

\section{The main theorem}

For $X, Y \subset G$ and $S<\Re(X), T \leqslant \Re(Y)$ the elements $\bar{X}_{S}^{G}$ and $\bar{Y}_{T}^{G}$ multiply according to the Mackey decomposition

$$
\bar{X}_{S}^{G} \bar{Y}_{T}^{G}=\sum_{g \in \Omega}\left(\bar{X} \bar{Y}^{g}\right)_{S \cap T^{*}}^{C}
$$

where $\Omega$ is a set of $(S, T)$ double coset representatives in $L$. For $S<K<G$, we have trivially that

$$
\left(\bar{X}_{S}^{K}\right)_{K}^{G}=\bar{X}_{S}^{G} .
$$

We first outline the proof of Theorem 1. It must be shown that if $(\bar{H} y)_{N}^{G} \in$ $\mho(L, n)$ and $b_{C}^{G}$ is a conjugacy class sum then their product lies in $\mho(L, n)$. By Eq. (1) this product is the sum of terms $(\bar{H} u)_{S}^{G}$ where $u=y b^{8}$ and $S=N \cap C^{g}$, which do not have the form required by the above spanning set of $\mathscr{W}(L, n)$. However we show [Lemma 31] that $H u$ may be partitioned into conjugates of cosets $H_{x} x, H_{x}$ being the maximum subgroup of $H$ normalized by $x$. This gives [Lemma 4] $\widetilde{H} u$ as the sum of terms $\left(\bar{H}_{x} x\right)_{T(x, u)}^{K}$ where $K=\Re(H u) \cap N$ and $T(x, u)=K \cap \Re\left(H_{x} x\right)$. From this and Eq. (2) we obtain $(\bar{H} u)_{S}^{G}$ as the sum of terms $\left(\vec{H}_{x} x\right)_{T(x, u)}^{G}$ which are shown to be in the given spanning set.

For $H<G$ and $u \in G, H_{u}$ denotes the unique maximal subgroup of $H$ which $u$ normalizes.

Lemma 1.

$$
H \cap \Re\left(H_{u} u\right) \stackrel{(\mathrm{a})}{=} H_{u} \stackrel{(\mathrm{b})}{<} H \cap H^{u} \stackrel{(\mathrm{c})}{=} H \cap \Re(H u \stackrel{(\mathrm{d})}{\triangleleft} \Re(H u) \stackrel{(\mathrm{e})}{<} \Re(H) .
$$

Proof. We verify the chain from the right. $x \in N(H u)$ implies $H u=H^{x} u^{x}$ and so $H u u^{-1} H=H^{x} u^{x}\left(u^{x}\right)^{-1} H^{x}$, that is $H=H^{x}$ proving (e). Trivially then $\Re(H u)$ normalizes $H \cap \Re(H u)$ giving (d). Also trivially $H \cap H^{u}<H \cap$ $\mathfrak{U}(H u)$. If $x \in H \cap \mathcal{N}(H u)$ then as above $u^{x} \in H u$ giving $x \in H^{u}$; so $H \cap$ $\Re(H u)<H \cap H^{u}$ giving (c). (b) is immediate from the definition of $H_{u}$. 
Trivially $H_{u} \leqslant H \cap \mathscr{R}\left(H_{u} u\right)$. If $h \in H \cap \Re\left(H_{u} u\right)$ then the inclusion $u^{h} \in$ $H_{u} u$ may be rewritten $u h u^{-1} \in h H_{u} \subset H \cap N\left(H_{u} u\right)$. So $u^{-1}$;nd hence $u$ normalize $H \cap \mathscr{N}\left(H_{u} u\right)$. By definition of $H_{u}, H \cap \mathfrak{N}\left(H_{u} u\right)<H_{u}$ proving (a).

Corollary. $x \in \mathcal{N}(H u)$ if and only if $x \in \mathcal{N}(H)$ and $\left[x, u^{-1}\right] \in H$.

Proof. Necessity is immediate from the proof of (e). If $\left[x, u^{-1}\right] \in H$ and $x \in \mathcal{N}(H)$ then $u^{x} \in H u$ and so $(H u)^{x}=H^{x} u^{x} \subset H H u=H u$.

Lemma 2. If $H \cap H^{u} \leqslant K \leqslant \Re(H u)$ then $K \cap \Re\left(H_{u} u\right)$ : $H_{u}$ divides $K H: H$.

Proof. From Lemma $1, H \cap H^{u} \triangleleft \Re(H u), H \cap K=H \cap H^{u}$ and $\{K \cap$ $\left.\Re\left(H_{u} u\right)\right\} \cap\left\{H \cap H^{u}\right\}=H_{u}$. So

$$
\frac{K \cap \mathscr{N}\left(H_{u} u\right)}{H_{u}} \cong \frac{\left\{K \cap \mathscr{N}\left(H_{u} u\right)\right\}\left\{H \cap H^{u}\right\}}{H \cap H^{u}}<\frac{K}{H \cap H^{u}} \simeq \frac{K H}{H} .
$$

Next we obtain a partition of the coset $H u$ into cosets of the form $H_{x} x$. First note that if $y \in H_{x} x$ then $H_{y}=H_{x}$ and so $H_{y} y=H_{x} x$; for $y$ normalizes $H_{x}$ giving $H_{x}<H_{y}$ and then $x \in H_{y} y$ giving $H_{y}<H_{x}$. The cosets $H_{x} x$, and $H_{y} y$ are thus either equal or disjoint and so we get a partition of $G$ into cosets of form $H_{x} x$.

Lemma 3. (a) The set $\mathscr{P}=\left\{H_{x} x, x \in G\right\}$ is a partition of $G$, permuted by conjugation by $\Re(H)$.

(b) The set $\mathscr{P}^{\prime}=\left\{H_{x} x, x \in H u\right\}$ is a partition of $H u$, permuted by conjugation by $\Re(H u)$.

Proof. For $g \in \mathfrak{X}(H), H_{x}^{g}=H_{z}$ where $z=x^{g}$. So $\left(H_{x} x\right)^{g}=H_{z} z \in \mathscr{P}$, proving (a). If $g \in \mathscr{N}(H u)$ then $H_{x} x \in \mathscr{P}^{\prime}$ implies $H_{z} z \in \mathscr{P}^{\prime}$, proving (b).

We can immediately obtain a decomposition of an arbitrary coset sum $\bar{H} u$.

LeMmA 4. Let $K \leqslant \Re(H u)$ and let $\left\{H_{x} x, x \in \Lambda(K)\right\}$ be a set of representatives of the distinct $K$-orbits of $\mathcal{P}^{\prime}$. Then

$$
\bar{H} u=\sum_{x \in \Lambda(K)}\left(\bar{H}_{x} x\right)_{K \cap \pi\left(H_{x} x\right)}^{K}
$$

The proof is trivial when it is noted that each summand is the sum of all the distinct cosets within a $K$-orbit. 
Proof of Theorem. Let $(\bar{H} y)_{N}^{G} \in \mathcal{W}(L, n)$, let $b_{C}^{G}$ be a conjugacy class sum and $\Omega$ a set of $(N, C)$ double coset representatives. By (1)

$$
\begin{aligned}
(\bar{H} y)_{N}^{G} b_{C}^{G} & =\sum_{g \in \Omega}\left(\bar{H} y b^{g}\right)_{N \cap C^{g}}^{G} \\
& =\sum_{u}\left(K: N \cap C^{g}\right)(\bar{H} u)_{K}^{G}
\end{aligned}
$$

where $u=y b^{g}$ and $K=\mathscr{R}(H u) \cap N$. By Lemma 4 and Eq. (2) $(\overline{H u})_{K}^{G}$ is the sum of terms $\left(\bar{H}_{x} x\right)_{T(x, u)}^{G}$ where $T(x, u)=K \cap \mathcal{K}\left(H_{x} x\right)$. We show that these terms lie in the given spanning set of $W(L, n)$. By definition we have that $H_{x}<H<L$ and $x \in \Re\left(H_{x}\right)$. Also $H_{x} \leqslant H \cap H^{x}=H \cap H^{u}=H \cap$ $\mathfrak{N}(H u)<N \cap \mathfrak{N}(H u)=K$; so $H_{x} \leqslant T(x, u) \leqslant \Re\left(H_{x} x\right)$. Finally since $T(x, u)=K \cap \mathfrak{R}\left(H_{x} x\right)$, by Lemma $2, T(x, u): H_{x}$ divides $K H: H$ which divides $N: H$ which divides $n$.

It may be noted that a slight generalization of Theorem 1 may be obtained by replacing $\Re(H y)$ by $\mathscr{X}(H y) \cap T$ where $L \leqslant T \leqslant G$.

\section{The modular case}

Extending the coefficient ring from $J$ to $R$, the ring of $p$-adic integers, gives ideals $\mathscr{W}_{R}(L, n)$ of $Z(R G)$. If $|G|$ is a unit in $R$ then $\mathscr{W f}_{R}(L, n)=Z(R G)$ for each conjugacy class sum may be written $\{b\}_{1}^{G} /|C(b)|$. However on passing from $R$ to $k$, the residue class field by the natural homomorphism, the ideals $W^{\prime}(L, n)$ of $Z(k G)$ so obtained are non-trivial when $p$ divides $|G|$. In this case we may restrict $n$ and $N$.

THEOREM 2. For $n=m p^{\alpha}$ and $(m, p)=1$ the ideal $\mathcal{W}^{\prime}(L, n)$ equals $W^{\prime}\left(L, p^{\alpha}\right)$ and is spanned by the set $\left\{(\bar{H} y)_{P}^{G} / H \leqslant L, y \in \Re(H), P\right.$ a Sylow p-subgroup of $\Re(H y), P: H \cap P$ divides $\left.p^{\alpha}\right\}$

Proof. Let $\beta=(\bar{H} y)_{N}^{G}\left(\in \mho^{\prime}(L, n)\right)$ and $P$ be a Sylow $p$-subgroup of $N$. Then $N: H P$ is a unit and $\beta=(\bar{H} y)_{H P}^{G} / N: H P$. Here $H P: H(=P: H \cap P)$ is the maximum power of $p$ dividing $N: H$ and so divides $p^{\alpha}$. So $W^{\prime}(L, n) \subset$ $W^{\prime}\left(L, p^{\alpha}\right)$ and trivially $W^{\prime}\left(L, p^{\alpha}\right) \subset \mathscr{W}^{\prime}(L, n)$. Since $(\bar{H} y)_{N}^{G}=(\bar{H} y)_{P}^{G} / N: P$, $\mathscr{W}^{\prime}(L, n)$ is spanned by the elements $(\bar{H} y)_{P}^{G}$, which are non-zero only if $P$ is a Sylow $p$-subgroup of $\mathfrak{T}(H y)$.

We now restrict further to the case where $L$ is a $p$-subgroup and obtain a further restriction of the spanning set. Let $y=r s=s r$ with $r p$-regular, $s$ a $p$-element, $P$ a subgroup of $L$ and $y \in \mathfrak{N}(P)$. 
LEMMA 5. $\Re(P y) \leqslant \Re(P r)$.

Proof. By the corollary to Lemma $1, x \in \mathfrak{R}(P y)$ if and only if $x \in \mathfrak{N}(P)$ and $x^{-1} y x y^{-1} \in P$, that is $y^{x} \in P y$. Since $r=y^{m}$ for some integer $m, r \in \mathfrak{X}(P)$ and $r^{x}=\left(y^{m}\right)^{x}=\left(y^{x}\right)^{m} \in P y^{m}=P r$.

Lemma 6. Let y normalize both $P$ and $Q=P_{0}<P$. Define recursively $P_{i+1}=$ $\mathfrak{X}\left(P_{i}\right) \cap P, i=0,1,2, \ldots$ Then $y \in \mathscr{N}\left(P_{i}\right)$ and $(\bar{Q} y)_{Q}^{P}=0$ if and only if for some $i$, $\mathscr{N}\left(P_{i} y\right) \cap P_{i+1}>P_{i}$. Otherwise $(\overline{Q y})_{Q}^{P}=\bar{P} y$.

Proof. For some $l, P_{l}=P$. The proof is by induction on the minimal such $l$. Since $y \in \mathscr{R}(Q)$ and $y$ normalizes $P, y$ normalizes $P \cap \mathscr{N}(Q)=P_{1}$. If $\mathscr{N}(Q y)$ $\cap P_{1}>Q$ then $(\overline{Q y})_{Q}^{P_{1}}=0$ whence $(\bar{Q} y)_{Q}^{P}=0$. Otherwise let $T$ be a transversal of $Q$ in $P_{1}$ and so

$$
(\bar{Q} y)_{Q}^{P_{1}}=\sum_{u \in T}(\bar{Q} y)^{u}=\sum_{u \in T} \bar{Q} y^{u} .
$$

Here $y^{u}=\left(u^{-1} y u y^{-1}\right) y=q_{u} y$ where $q_{u}=u^{-1}\left(y u y^{-1}\right) \in P_{1} . q_{u} \in Q q_{v}$ implies $y^{u}$ $\in Q y^{v}$, that is $u v^{-1} \in \mathcal{U}(Q y) \cap P_{1}=Q$. So the cosets $Q q_{u}, u \in T$, are distinct and $(\bar{Q} y)_{Q}^{P_{1}}=\Sigma_{u \in T} \bar{Q} q_{u} y=\bar{P}_{1} y$. Applying the hypothesis to the chain from $P_{1}$ to $P$, we have the result.

Let $N=\Re(P y) \leqslant \Re(P)$; then $y, r$, and $s \in N$. Let $C=C(r) \cap N$ and $Q=P \cap C$. Let $D$ be a Sylow $p$-subgroup of $C$. Then $C$ and hence $D$ normalize $Q$ and so $D \leqslant \Re(Q r)$. Further by the corollary to Lemma 1 since $D<\Re(P y)$ we have $y y^{-1} \in P$ for all $d \in D$; trivially also $y y^{d}=d^{-1}\left(y d y^{-1}\right) \in C$ and so $y^{d} y^{-1} \in P \cap C=Q$. So by the same corollary, $D<\Re(Q y)$.

LeMMA 7. $(\bar{Q} y)_{D}^{N}=(N: P D) \bar{P} y \neq 0$.

Proof. $(\bar{Q} r)_{D}^{N}$ is the sum of $N$-conjugacy classes, the only $p$-regular class term being $r_{D}^{N}=(C: D) r_{C}^{N} \neq 0$. So $(\bar{Q} r)_{D}^{N} \neq 0$. In particular $(\bar{Q} r)_{D}^{P D} \neq 0$. Since a transversal of $D$ in $P D$ is a transversal of $Q$ in $P$, we have $(\overline{Q r})_{D}^{P D}=(\bar{Q} r)_{Q}^{P}$; and so by Lemma $6 \mathscr{N}\left(P_{i} r\right) \cap P_{i+1}=P_{i}$ and $(\bar{Q} r)_{D}^{N}=(\bar{P} r)_{P D}^{N}=(N: P D) \bar{P} r$. Thus $P D$ is a Sylow $p$-subgroup of $N$. Since $\Re\left(P_{i} y\right) \cap P_{i+1}<\Re\left(P_{i} r\right) \cap P_{i+1}=P_{i}$, again by Lemma 6, we have $(\bar{Q} y)_{Q}^{P}=\bar{P} y$ and so $(\bar{Q} y)_{P}^{N}=(N: P D) \bar{P} y \neq 0$, as required.

THEOREM 3. Let $L$ be a p-subgroup of $G$. Then $\mathcal{W} S^{\prime}\left(L, p^{\alpha}\right)$ is spanned by the set $\left\{(\bar{P} y)_{D}^{G} / P<D<C(r) \cap L, r\right.$ the p-regular part of $y \in \mathcal{X}(P), D$ a Sylow p-subgroup of $\Re(P y), D: P$ divides $\left.p^{\alpha}\right\}$. 
The proof is an immediate consequence of Lemma 7 since an arbitrary generator $(\bar{P} y)_{N}^{G}$ of $\mho^{\prime}\left(L, p^{\alpha}\right)$ is a non-zero multiple of $(\bar{Q} y)_{D}^{G}$ which lies in the above set.

We conclude by noting that when $\alpha=0$ and $L$ is a Sylow p-subgroup of $G$, the elements of the above spanning set are of the form $(\bar{D} y)_{D}^{G}=(\bar{D} r)_{D}^{G}$ since $s \in D$. But these elements e just the $p^{\prime}$-section sums of Lemma 2 in O'Reilly (1973), giving the ideal of Reynolds (1972) Theorem 1. This ideal has also been studied in Broué (1978) and lizuka (1973).

If $L$ is a Sylow $p$-subgroup of $G, \mathscr{w}^{\prime}\left(L, p^{\alpha}\right)$ will contain only those $p$-regular classes, and hence block idempotents, of defect $<p^{\alpha}$.

\section{References}

M. Broué (1978) 'Radicals, hauteurs, $p$-sections et blocs', Ann. of Math. 107, 89-107.

K. Iizuka, Y. Ito, A. Watanabe (1973), 'A remark on the representations of finite groups IV, Memoirs of the Faculty of General Education, Kumamato Univ., Natural Science Series, 8, 1-5.

M. F. O'Reilly (1973) 'Ideals in the centre of a group ring', Proc. Second Internat. Conf. Theory of Groups, pp. 536-540 (Lecture Notes in Mathematics 372, Springer Verlag, Berlin).

W. F. Reynolds (1972) 'Sections and ideals of centers of group algebras', Algebra 20, 176-181.

Department of Mathematics

Rhodes University

Grahamstown, 6140

South Africa 